\title{
The effect of varus knee deformities on the ankle alignment in patients with knee osteoarthritis
}

\author{
Kai Xie ${ }^{1 \dagger}$, Xuequan $\mathrm{Han}^{1 \dagger}, \mathrm{Xu}$ Jiang ${ }^{1 \dagger}$, Songtao $\mathrm{Ai}^{2}$, Kerong Dai ${ }^{1}$, Zhifeng $\mathrm{Yu}^{1}$, Haishan $\mathrm{Wu}^{1}$, Xinhua $\mathrm{Qu}^{3^{*}}$ and \\ Mengning $\operatorname{Yan}^{1^{*}}$ (D)
}

\begin{abstract}
Background: We evaluated the compensatory change in ankle alignment due to knee malalignment and its relationship with varus knee deformities, as well as sex differences in compensation.

Methods: From October 2016 to September 2017, 103 patients with end-stage knee osteoarthritis underwent primary total knee arthroplasty (TKA). Ninety-five knees (78 patients) were included. The hip-knee-ankle angle (HKA) and ankle alignment and tilt were evaluated with full-leg standing anteroposterior radiographs. The ankle alignment was estimated according to the tibiotalar angle, tibial anterior surface angle (TAS), and lateral distal tibial angle. The talar tilt angle (TT), anatomical talocrural angle, angle between the tibial plateau and distal tibial plafond, angles between the ground and distal tibial plafond, and angles between the ground and upper talus were measured to evaluate ankle tilt. The patients were separated into two sex-based groups; correlations between the HKA and ankle parameters were estimated.

Results: The mean HKA in men and women was $8.16 \pm 4.36^{\circ}$ and $7.69 \pm 5.93^{\circ}$, respectively. The relative tilt of the talus and distal tibia plafond to the ground was increased when varus knee deformities progressed. In women, there was a positive correlation between the knee alignment and TAS $(r=-0.295, p=0.016)$. As the knee mechanical axis became more varus, the distal tibia plafond became more valgus. In women, a negative correlation was observed between the HKA and $\Pi(r=-0.359, p=0.003)$. Compensatory changes in the ankle alignment and $\Pi$ to knee alignment were not observed in men.
\end{abstract}

Conclusion: Compensatory ankle changes should be considered before TKA.

Keywords: Total knee arthroplasty, Knee alignment, Ankle malalignment, Varus knee deformity

\section{Background}

Osteoarthritis (OA) is a major chronic disease worldwide, affecting more than $10 \%$ of people over 60 years old with joint pain and disability [1]. The knee is a complex joint that bears a high load during activities of daily life. Inappropriate joint biomechanics are a main risk

\footnotetext{
*Correspondence: xinhua_qu@126.com; yanmengning@163.com ${ }^{\dagger}$ Kai Xie, Xuequan Han, and Xu Jiang contributed equally to this work. ${ }^{3}$ Department of Bone and Joint Surgery, Renji Hospital, School of Medicine, Shanghai Jiao Tong University School of Medicine, 145 Middle Shandong Road, Shanghai, China

${ }^{1}$ Shanghai Key Laboratory of Orthopaedic Implants, Department of Orthopaedic Surgery, Shanghai Ninth People's Hospital, Shanghai Jiao Tong University School of Medicine, 639 Zhizaoju Road, Shanghai, China Full list of author information is available at the end of the article
}

factor of knee OA [2]. The joint biomechanics are directly affected by lower extremity malalignment caused by an anatomical deformity. The mechanical alignment of the lower extremity is highly determined by the morphology of the hip, knee, and ankle joints. Varus deformities of the knee cause overloading and cartilage wear in the medial compartment, which could exacerbate degeneration of the knee joint. Severe knee OA is a long-term pathological process that could change the alignment of the entire lower limb and accelerate degeneration of the ankle joint [3].

Recently, ankle OA has received more attention from orthopedists than it has in the past; however, the prevalence of symptomatic ankle OA is still unknown.

(c) The Author(s). 2019 Open Access This article is distributed under the terms of the Creative Commons Attribution 4.0 International License (http://creativecommons.org/licenses/by/4.0/), which permits unrestricted use, distribution, and 
Trauma is the most prevalent etiology of ankle OA, and posttraumatic OA accounts for approximately $70 \%$ of ankle OA cases [4-6]. The prevalence of primary ankle $\mathrm{OA}$ is rare, whereas $35.2 \%$ of patients with knee OA have a radiologic degenerative change in the ankle before undergoing total knee arthroplasty (TKA) [7]. The ankle would respond to varus knee deformities as an important part of lower extremity alignment. A few previous studies have reported that radiologic malalignment occurs in both the ankle and hindfoot joints secondary to varus knee deformities [7-9]. Valgus ankle alignment and a lateral metaphyseal, collapsed, distal tibia were observed in patients with varus knee deformities. For hindfoot alignment, an increase in varus knee deformities would make the hindfoot alignment more valgus. However, no evidence supports the idea that compensatory changes in the ankle alignment would increase with the aggravation of varus knee deformities. It is unclear whether compensatory changes in the ankle alignment differ based on sex. The objectives of the current retrospective study were to estimate the change in ankle alignment to compensate for varus knee deformities and evaluate differences in sex regarding these compensatory changes.

\section{Methods}

\section{Patient enrollment}

The current study was approved by our institutional review board (SH9H-2018-179-T137). Informed consent was obtained from all individual participants included in the study. Between October 2016 and September 2017, 103 patients with end-stage knee OA underwent primary TKA at our center. All patients underwent full-leg standing anteroposterior (AP) radiography before TKA. The inclusion criteria were TKA patients with radiologically seen knee OA and joint pain and/or dysfunction. The exclusion criteria were inadequate or missing AP radiographs, history of ipsilateral lower extremity surgery before TKA, and valgus knee alignment before TKA. Twenty-five patients were excluded (Table 1).

Ninety-five knees in 78 patients were enrolled. Twenty-four patients (29 knees) were men, and 54 (66 knees) were women. The mean age of the patients was 68.7 years (range 53-85). Seventeen patients underwent bilateral TKA, 39 patients underwent right-side TKA, and 22 patients underwent left-side TKA. The knee and ankle on the surgical side were evaluated in the following radiographic assessments.

\section{Radiographic assessment}

Patients underwent full-leg standing digital AP radiographs with the patellae forward and in full knee extension to avoid measuring errors caused by lower extremity rotation as previously described [10]. Digital radiographs were acquired using Kodak DirectView (Kodak, Rochester, NY, USA) from all patients before they underwent TKA, and stored in the General Electric system (General Electric, Boston, MA, USA). The level of knee $\mathrm{OA}$ was rated according to the Kellgren-Lawrence radiographic classification of OA [11, 12]. Materialise Mimics version 20.0 (Materialise, Leuven, Belgium) was used to perform all measurements. The hip-knee-ankle angle (HKA), tibiotalar angle (TTA), tibial anterior surface angle (TAS), lateral distal tibial angle (LDTA), talar tilt angle (TT), anatomical talocrural angle (TC), angle between the tibial plateau and distal tibial plafond (PP), angles between the ground surface and distal tibial plafond (GP), and angles between the ground surface and upper talus (GT) were measured using full-leg standing digital AP radiographs (Fig. 1).

Varus knee deformities were evaluated with the HKA, while tibiotalar joint alignment was evaluated with the TTA, as previously reported $[4,8]$. The femur mechanical axis was defined as a line from the center of the femoral head to the center of the knee. The tibia mechanical axis was defined as a line from the center of the tibial plateau to the center of the ankle. The HKA was defined as the angle between the mechanical axes of the femur and tibia. A HKA greater than or equal to $3^{\circ}$ was defined as varus knee deformity [13]. The TTA was defined as the angle between the tibia anatomical axis and talus upper surface.

The TAS and LDTA were used to evaluate compensatory changes in the distal tibial plafond. The TAS was defined as the angle between the tibia anatomical axis and distal tibial plafond. The LDTA was defined as the lateral angle between the tibia mechanical axis and distal tibial plafond. The tilt of the talus relative to the distal tibial plafond and ground was evaluated with the TT and GT. The TT was defined as the angle between the distal

Table 1 Exclusions from the whole cohort

\begin{tabular}{lc}
\hline Reason for exclusion & Number of patients \\
\hline Inadequate AP radiograph & 3 \\
Missing AP radiograph & 5 \\
History of ipsilateral lower extremity surgery before TKA & 4 \\
Valgus knee alignment before TKA & 13 \\
Total & 25 \\
\hline
\end{tabular}




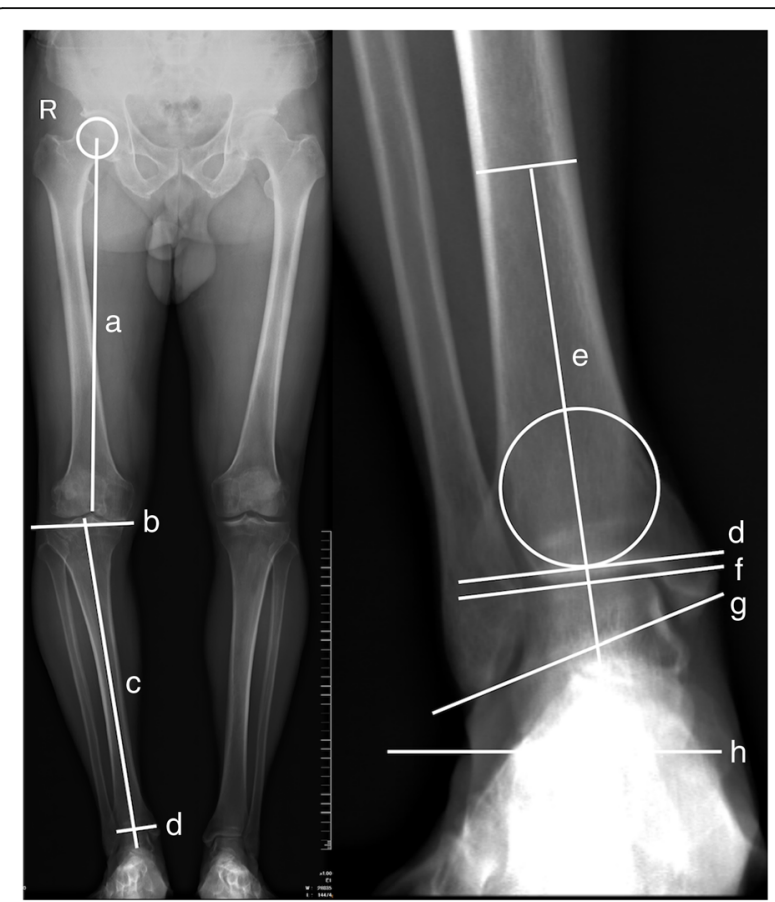

Fig. 1 Measurement of HKA and ankle parameters based on full-leg standing anteroposterior radiographs. TTA was defined as the medial angle between " $\mathrm{e}$ " and "f." TAS was defined as the medial angle between "e" and "d." LDTA was defined as the lateral angle between " $\mathrm{C}$ " and "d." " $T$ was defined as the angle between " $\mathrm{d}$ " and "f." GT was defined as the angles between "f" and "h." GP was defined as the angle between " $\mathrm{d}$ " and "h." PP was defined as the angles between "b" and "d." TC was defined as the medial angles between "e" and "g." Line a was the line from the center of the femoral head to the center of the knee. Line $b$ was the joint surface of the tibial plateau. Line $\mathrm{c}$ was the line from the center of the tibial plateau to the center of ankle. Line $d$ was the distal tibial plafond. Line e was the anatomical axis of the tibia. Line $f$ was the upper joint surface of the talus. Line $g$ was the line from medial malleolus to lateral malleolus. Line $h$ was the ground surface

tibia plafond and the upper joint surface of the talus. For the TT, an angle opening at the medial side was defined as a positive value. In the software that was used, a horizontal line indicated the ground surface. The GT was defined as the angle between the ground surface and upper talus, which was evaluated using the angle between a horizontal line and the upper talus. The TC was defined as the angle between the anatomical axis and a line from the medial malleolus to the lateral malleolus. The relative tilt of the talus upper joint surface to the tibial plateau and ground was evaluated with the PP and GP. The correlation between the HKA angle and ankle parameters was estimated.

\section{Statistical analysis}

SPSS version 23.0 (IBM Corp., Armonk, NY, USA) was used to perform all statistical analyses. A $p$ value less than 0.05 was considered statistically significant. The correlation between the HKA and other ankle parameters was evaluated with scatter plots and a two-tailed Pearson's correlation test. A two-tailed $t$ test was used to compare the HKA and ankle parameters in male and female patients.

\section{Results}

According to the Kellgren-Lawrence radiographic classification of OA, 20 knees had grade 3 and 75 knees had grade 4 . The mean HKA in grade 4 group was $9.05 \pm$ $5.43^{\circ}$, which was significantly higher than that in the grade 3 group $\left(3.26 \pm 2.38^{\circ}\right)$. As presented in Table 2, there were significant differences in ankle morphology (TAS, TT, GP, GT, and TC) between the grade 3 and grade 4 groups, indicating that varus knee deformity had a significant influence on ankle alignment in knee OA patients.

There was no statistically significant difference in the mean HKA and ankle parameters between men and women (Table 3). The correlation between the HKA and ankle parameters is presented in Table 4. The mean HKA in men and women was $8.16 \pm 4.36^{\circ}$ and $7.69 \pm$ $5.93^{\circ}$, respectively. The mean TTA in men and women were $89.84 \pm 3.35^{\circ}$ and $90.77 \pm 3.03^{\circ}$, respectively. The varus-to-normal tibiotalar joint alignment was observed in patients in both groups, and no statistically significant correlation was observed between the knee alignment and TTA. However, a significant correlation was observed between the knee alignment and TAS in women. In these patients, as the knee mechanical axis became more varus, the distal tibia plafond became more valgus $(r=0.295, p=0.016)$. The mean TAS in men and women was $89.10 \pm 3.49^{\circ}$ and $88.03 \pm 3.07^{\circ}$, respectively. The lateral metaphyseal collapse of the distal tibia plafond was

Table 2 Characteristics of patients with different grade of knee $\mathrm{OA}$

\begin{tabular}{llll}
\hline Characteristic & Grade 3 & Grade 4 & $p$ \\
\hline Number of patients & 18 & 60 & $/$ \\
Number of knees & 20 & 75 & $/$ \\
Mean age (year) & $68.10 \pm 6.03$ & $68.80 \pm 6.26$ & 0.439 \\
Disease duration & $6.60 \pm 5.86$ & $7.34 \pm 4.36$ & 0.363 \\
HKA & $3.26 \pm 2.38$ & $9.06 \pm 5.43$ & $<0.001$ \\
TTA & $88.86 \pm 3.34$ & $89.69 \pm 3.09$ & 0.296 \\
TAS & $87.06 \pm 3.53$ & $88.70 \pm 3.07$ & 0.043 \\
LDTA & $92.97 \pm 3.39$ & $91.57 \pm 2.82$ & 0.062 \\
TT & $1.97 \pm 0.92$ & $1.24 \pm 1.55$ & 0.047 \\
Ground-plafond (GP) & $7.37 \pm 3.42$ & $9.28 \pm 3.84$ & 0.046 \\
Ground-talus (GT) & $5.94 \pm 3.10$ & $8.12 \pm 4.24$ & 0.033 \\
Plateau-plafond (PP) & $7.11 \pm 4.08$ & $6.49 \pm 3.75$ & 0.514 \\
TC & $75.20 \pm 4.66$ & $77.48 \pm 3.40$ & 0.016 \\
\hline
\end{tabular}


Table 3 Characteristics of patients

\begin{tabular}{llll}
\hline Characteristic & Male & Female & $p$ \\
\hline Number of patients & 24 & 54 & $/$ \\
Number of knees & 29 & 66 & $/$ \\
Right/left & $18 / 11$ & $38 / 28$ & $/$ \\
Mean age (year) & $69.83 \pm 6.329$ & $68.17 \pm 6.25$ & 0.124 \\
HKA & $8.16 \pm 4.36$ & $7.69 \pm 5.93$ & 0.439 \\
TTA & $89.84 \pm 3.35$ & $90.77 \pm 3.03$ & 0.363 \\
TAS & $89.10 \pm 3.49$ & $88.03 \pm 3.07$ & 0.702 \\
LDTA & $91.11 \pm 2.93$ & $92.19 \pm 2.97$ & 0.186 \\
TT & $1.34 \pm 1.57$ & $1.42 \pm 1.43$ & 0.136 \\
Ground-plafond (GP) & $8.44 \pm 3.72$ & $9.07 \pm 3.88$ & 0.107 \\
Ground-talus (GT) & $7.19 \pm 4.06$ & $7.87 \pm 4.14$ & 0.817 \\
Plateau-plafond (PP) & $6.12 \pm 3.34$ & $6.84 \pm 4.00$ & 0.463 \\
TC & $77.43 \pm 3.76$ & $76.82 \pm 3.82$ & 0.460 \\
\hline
\end{tabular}

HKA hip-knee-ankle angle, TTA tibiotalar angle, TAS tibial anterior surface angle, LDTA lateral distal tibial angle, $\pi$ talar tilt angle, $T C$ anatomical talocrural angle, $P P$ angle between the tibial plateau and distal tibial plafond $G P$ angle between the ground surface and distal tibial plafond, $G T$ angle between the ground surface and upper talus

Table 4 The correlation of HKA with ankle parameters in male and female group

\begin{tabular}{lll}
\hline Ankle parameter & Correlation & $p$ \\
\hline Male & & \\
TTA & 0.154 & 0.425 \\
TAS & 0.246 & 0.199 \\
LDTA & -0.065 & 0.739 \\
$\Pi$ TT & -0.177 & 0.360 \\
Ground-plafond (GP) & 0.455 & 0.013 \\
Ground-talus (GT) & 0.476 & 0.009 \\
Plateau-plafond (PP) & 0.191 & 0.321 \\
TC & -0.001 & 0.994 \\
Female & & \\
TTA & 0.130 & 0.297 \\
TAS & 0.295 & 0.016 \\
LDTA & -0.219 & 0.077 \\
TT & -0.359 & 0.003 \\
Ground-plafond (GP) & 0.683 & $<0.001$ \\
Ground-talus (GT) & 0.751 & $<0.001$ \\
Plateau-plafond (PP) & 0.331 & 0.007 \\
TC & 0.203 & 0.103 \\
\hline
\end{tabular}

HKA hip-knee-ankle angle, TTA tibiotalar angle, TAS tibial anterior surface angle, LDTA lateral distal tibial angle, $\pi$ talar tilt angle, $T C$ anatomical talocrural angle, $P P$ angle between the tibial plateau and distal tibial plafond, $G P$ angle between the ground surface and distal tibial plafond, $G T$ angle between the ground surface and upper talus observed based on the measurement of the LDTA. The mean LDTAs in men and women were 91.11 $\pm 2.93^{\circ}$ and $92.19 \pm 2.97^{\circ}$, respectively. In women, although statistically insignificant, the LDTA decreased when the knee alignment became varus $(r=-0.219, p=0.077)$ (Fig. 2).

Compensatory change in the ankle tilt was observed in both men and women (Fig. 3). The TT in men and women was $1.34 \pm 1.57^{\circ}$ and $1.42 \pm 1.43^{\circ}$, respectively. A negative correlation was observed between the HKA and TT $(r=-0.359, p=0.003)$, confirming that there were compensatory changes in the ankle to a varus knee deformity. However, there was no correlation between the knee alignment and TT in men. The mean GT in men and women were $7.19 \pm 4.06^{\circ}$ and $7.87 \pm 4.14^{\circ}$, respectively. The mean GP in men and women were $8.44 \pm$ $3.72^{\circ}$ and $9.07 \pm 3.88^{\circ}$, respectively. Varus knee deformities had a significant influence on the GT $(r=0.751, p<$ $0.001)$ and GP $(r=0.683, p<0.001)$ angles in women. In men, the HKA was moderately and positively correlated with the GT $(r=0.455, p=0.009)$ and GP $(r=0.455, p<$ 0.013). In women, because the lateral metaphyses of the distal tibia plafond and tibial plateau collapsed, the angle between the tibial plateau and distal tibial plafond was also increased as the knee became more varus $(r=0.331$, $p=0.007)$. The mean $\mathrm{TC}$ in men and women was 77.43 $\pm 3.76^{\circ}$ and $76.82 \pm 3.82^{\circ}$, respectively. There was no correlation between the TC and HKA.

\section{Discussion}

The objectives of the current retrospective study were to estimate the change in ankle alignment to compensate for varus knee deformities and evaluate differences in sex regarding these compensatory changes. In women, the ankle alignment was significantly influenced by varus knee deformities, and as the knee mechanical axis became more varus, the distal tibia plafond secondarily became more valgus. However, a correlation between changes in the ankle alignment and knee mechanical axis was not found in male patients. Moreover, we found that abnormal biomechanics in the ankle were significantly associated with varus knee deformities in both men and women.

Although the effect of varus knee deformities on degenerative changes in the ankle has not been fully established, the relationship between varus knee deformities and ankle OA has been proven [14]. The most prevalent type of ankle OA is posttraumatic OA, while the prevalence of primary and secondary ankle OA is rarely reported. However, as previously described, the prevalence of ankle OA ranges from 28.8 to $35.2 \%$ in TKA patients without a history of ankle fracture [7, 15]. In a study on cadaveric donors, severe ankle degeneration always existed along with knee degeneration [16]. 

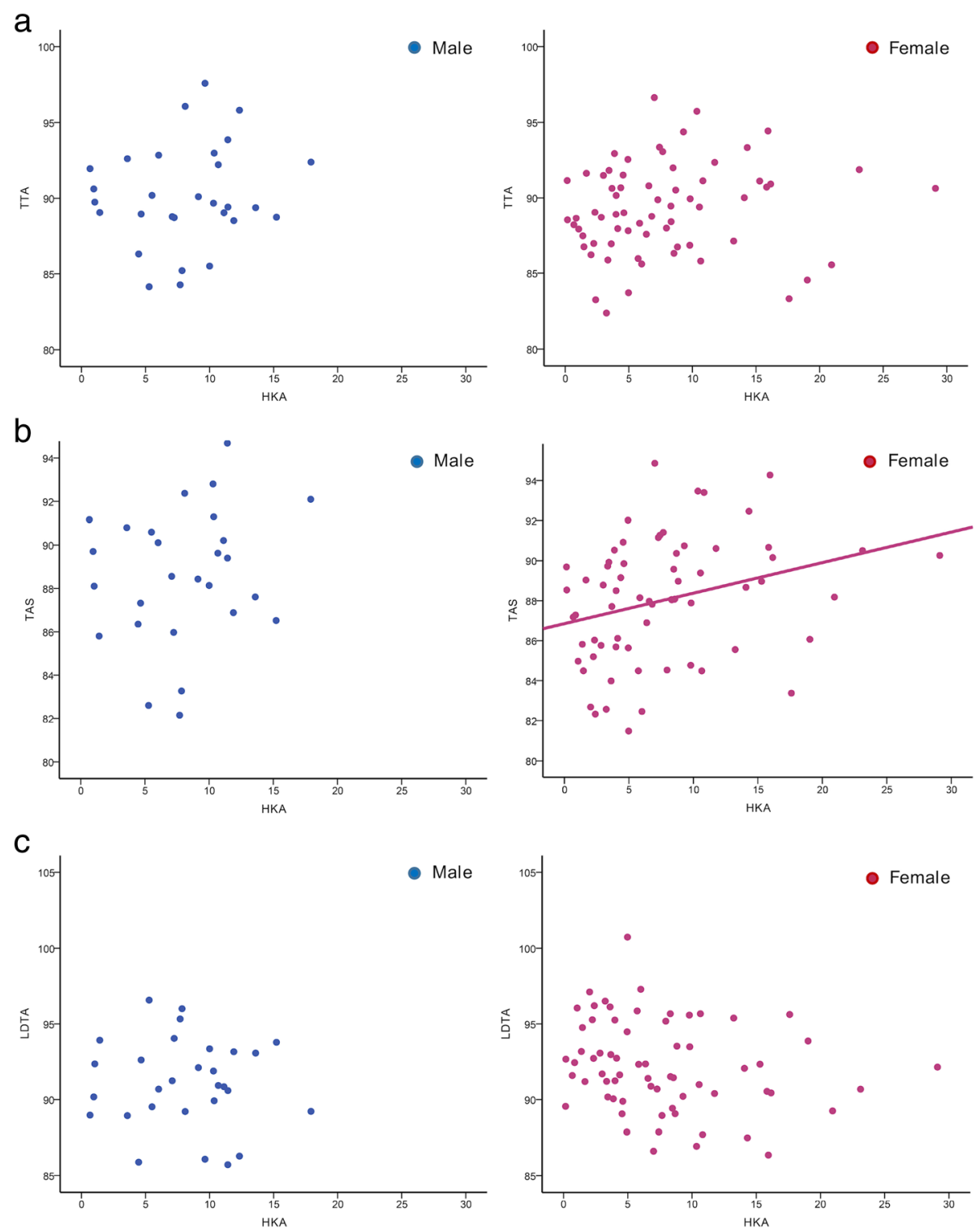

Fig. 2 The correlation analysis between HKA and compensatory ankle change (a TTA, b TAS, and $\mathbf{c}$ LDTA.The scatter diagram indicated a correlation between the HKA and TAS in female group (b)

Degeneration of the lower limb joints is highly related to the joint alignment. In the current study, the LDTA decreased as the knee alignment became more varus, indicating lateral metaphyseal collapse and valgus change in the distal tibia plafond. Our results show that compensatory changes in the ankle to varus knee deformities mainly occur at the distal tibial side, which could influence the ankle tilt and cause an abnormal biomechanical state in the tibiotalar joint. Therefore, we believe that valgus change in the distal tibial plafond and increased ankle tilt could be the main causes of ankle degeneration due to varus knee deformities.

Another interesting finding of the current study is that compensatory change in the ankle tilt to varus knee deformities could increase abnormal forces on the ankle.
In women, the TT decreased as the knee alignment became more varus, indicating lateral tilt of the tibiotalar joint. In addition, the relative tilt of the talus and distal tibia plafond to the ground also increased as varus knee deformities progressed. Increased tilt of the talus and distal tibia plafond could change the biomechanical state of the ankle, which could lead to narrowing of the medial ankle joint space and collapse of the lateral metaphysis of the distal tibia plafond. It is still uncertain whether the compensatory change in ankle morphology could recover after TKA. Therefore, it is necessary to evaluate the ankle alignment in knee OA patients preoperatively and postoperatively, which could further confirm the effect of varus knee deformity on the ankle alignment. 

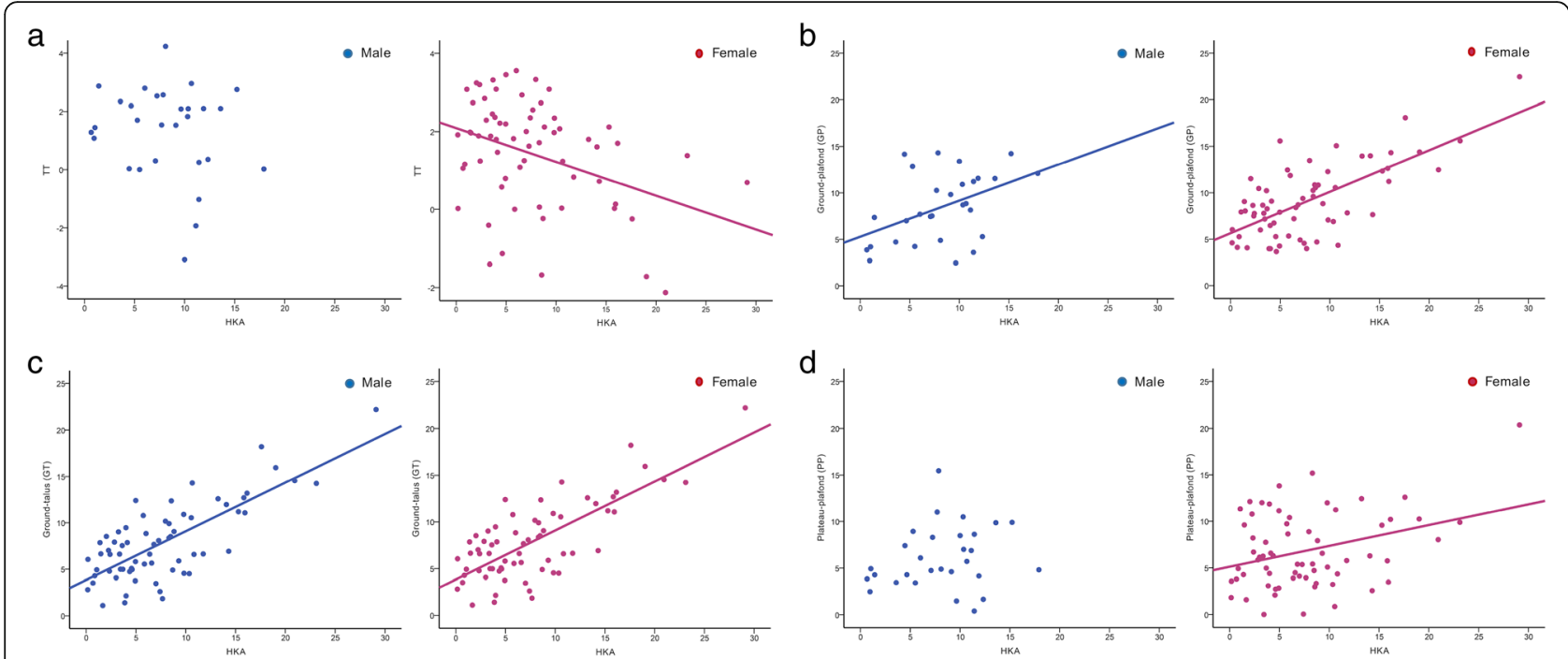

Fig. 3 The correlation analysis between the HKA and tilt of ankle (a TT, b GP, c GT, $\mathbf{d} P P)$ in both male and female patients

A more perplexing area of uncertainty is whether the compensatory change in ankle morphology negatively affects the outcome of TKA. TKA is the most cost-effective method to treat end-stage knee degeneration [17]. In past decades, the surgical techniques, perioperative management, and prosthesis survivorship of TKA have improved dramatically, but the patient-reported dissatisfaction rate of TKA ranges from 11 to $25 \%$ [18, 19]. Many factors could affect the patient's satisfaction with TKA, such as age, preoperative mental health, and postoperative knee stability $[20,21]$. In recent years, the effects of ankle and foot stability on TKA outcomes have received more attention. Gursu et al. [9] evaluated the ankle morphology and alignment in 80 knees with at least $10^{\circ}$ of varus deformities and concluded that overcorrecting the tilt of the distal tibia plafond and ankle alignment could be the main reason for postoperative ankle pain. As mentioned before, in women, lateral tilt of the distal tibia plafond and valgus changes to it would increase when the knee alignment becomes more varus. In such patients, leaving a residual varus knee deformity may lead to a better clinical outcome, which corresponds to our clinical experience. For most arthroplasty surgeons, it is uncommon to estimate the alignment and abnormal biomechanical state of the ankle before TKA. Based on the results of the current study and previous articles, we suggest performing a careful preoperative examination for ankle deformities in patients with knee OA, especially women with large varus knee deformities.

The current study has some limitations. First, the number of men was not large due to the relatively low prevalence of TKA in the male population. To our knowledge, we are the first to report sex differences in terms of compensatory change in the ankle alignment due to varus knee deformities. Moreover, the findings of the current study could provide a new perspective for further research. Second, the anatomic data that were used were from the Asian population, and the results of this study may not be generalized to people of other ethnicities. Third, we did not investigate the influence of hand dominance and patient complaints (such as joint pain and range of motion) on compensatory change in ankle alignment due to knee malalignment.

\section{Conclusions}

We observed a significant correlation between ankle malalignment and varus knee deformity in patients with knee OA. Compensatory changes in the ankle should be considered before TKA is performed. More large-scale and well-designed research studies are needed to fully demonstrate the effect of knee malalignment on ipsilateral and contralateral ankle alignment.

\section{Abbreviations}

AP: Anteroposterior; GP: Angle between the ground surface and distal tibial plafond; GT: Angle between the ground surface and upper talus; HKA: Hipknee-ankle angle; LDTA: Lateral distal tibial angle; OA: Osteoarthritis;

PP: Angle between the tibial plateau and distal tibial plafond; TAS: Tibial anterior surface angle; TC: Anatomical talocrural angle; TKA: Total knee arthroplasty; T: Talar tilt angle; TTA: Tibiotalar angle

\section{Acknowledgements}

Not applicable.

\section{Funding}

This study was supported by the Project of the Shanghai Collaborative Innovation Center for Translational Medicine (Grant No. TM201814), Clinical Research Program of 9th People's Hospital affiliated to Shanghai Jiao Tong Univeristy School of Medicine (Grant No. JYL025), Technology and Innovation Fund (Chuang Ke, Grant No. CK2018011), National Natural Science Foundation of China (Grant No. 11572197, 81401852, and 51631009), "Chen Guang" Project of the Shanghai Municipal Education Commission, and Shanghai Education Development Foundation (Grant No. 14CG14). 


\section{Availability of data and materials}

The datasets used and/or analyzed during the current study are available from the corresponding author on reasonable request.

\section{Authors' contributions}

$\mathrm{KX}, \mathrm{XH}$, and $\mathrm{XJ}$ participated in the data collection and evaluation. SA and ZY participated in the data analysis. KD, XQ, and MY participated in the study design. HW participated in the manuscript revision. All authors participated in the manuscript preparation and approved the final manuscript.

\section{Ethics approval and consent to participate}

The current retrospective study was approved by the institutional review board of Shanghai Ninth People's Hospital, Shanghai Jiao Tong University School of Medicine (SH9H-2018-179-T137). The informed consent was obtained for each patient.

\section{Consent for publication}

Not applicable.

\section{Competing interests}

The authors declare that they have no competing interests.

\section{Publisher's Note}

Springer Nature remains neutral with regard to jurisdictional claims in published maps and institutional affiliations.

\section{Author details}

'Shanghai Key Laboratory of Orthopaedic Implants, Department of Orthopaedic Surgery, Shanghai Ninth People's Hospital, Shanghai Jiao Tong University School of Medicine, 639 Zhizaoju Road, Shanghai, China. ${ }^{2}$ Department of Radiology, Shanghai Ninth People's Hospital, Shanghai Jiao Tong University School of Medicine, 639 Zhizaoju Road, Shanghai, China. ${ }^{3}$ Department of Bone and Joint Surgery, Renji Hospital, School of Medicine, Shanghai Jiao Tong University School of Medicine, 145 Middle Shandong Road, Shanghai, China.

\section{Received: 5 June 2018 Accepted: 3 May 2019}

Published online: 15 May 2019

\section{References}

1. Glyn-Jones S, Palmer AJ, Agricola R, Price AJ, Vincent TL, Weinans H, et al. Osteoarthritis. Lancet. 2015;386(9991):376-87.

2. Saxby DJ, Lloyd DG. Osteoarthritis year in review 2016: mechanics. Osteoarthritis Cartilage. 2017;25(2):190-8.

3. Hubbard TJ, Hicks-Little C, Cordova M. Changes in ankle mechanical stability in those with knee osteoarthritis. Arch Phys Med Rehabil. 2010;91(1):73-7.

4. Valderrabano V, Horisberger M, Russell I, Dougall H, Hintermann B. Etiology of ankle osteoarthritis. Clin Orthop Relat Res. 2009;467(7):1800-6.

5. Brown TD, Johnston RC, Saltzman CL, Marsh JL, Buckwalter JA. Posttraumatic osteoarthritis: a first estimate of incidence, prevalence, and burden of disease. J Orthop Trauma. 2006;20(10):739-44.

6. Saltzman CL, Salamon ML, Blanchard GM, Huff T, Hayes A, Buckwalter JA, et al. Epidemiology of ankle arthritis: report of a consecutive series of 639 patients from a tertiary orthopaedic center. lowa Orthop J. 2005;25:44-6.

7. Lee $\mathrm{JH}$, Jeong BO. Radiologic changes of ankle joint after total knee arthroplasty. Foot Ankle Int. 2012;33(12):1087-92

8. Gao F, Ma J, Sun W, Guo W, Li Z, Wang W. The influence of knee malalignment on the ankle alignment in varus and valgus gonarthrosis based on radiographic measurement. Eur J Radiol. 2016;85(1):228-32.

9. Gursu S, Sofu H, Verdonk P, Sahin V. Effects of total knee arthroplasty on ankle alignment in patients with varus gonarthrosis: do we sacrifice ankle to the knee? Knee Surg Sports Traumatol Arthrosc. 2016;24(8):2470-5.

10. Pinsornsak P, Naratrikun K, Kanitnate S, Sangkomkamhang T. The one-leg standing radiograph: an improved technique to evaluate the severity of knee osteoarthritis. Bone Joint Res. 2016;5(9):436-41.

11. Kellgren JH, Lawrence JS. Radiological assessment of osteo-arthrosis. Ann Rheum Dis. 1957:16(4):494-502

12. Kohn MD, Sassoon AA, Fernando ND. Classifications in brief: KellgrenLawrence classification of osteoarthritis. Clin Orthop Relat Res. 2016;474(8): 1886-93.
13. Rames RD, Mathison M, Meyer Z, Barrack RL, Nam D. No impact of undercorrection and joint line obliquity on clinical outcomes of total knee arthroplasty for the varus knee. Knee Surg Sports Traumatol Arthrosc. 2018; 26(5):1506-14.

14. Xie K, Jiang X, Han X, Ai S, Qu X, Yan M. Association between knee malalignment and ankle degeneration in patients with end-stage knee osteoarthritis. J Arthroplasty. 2018;33(12):3694-8 e1.

15. Tallroth K, Harilainen A, Kerttula L, Sayed R. Ankle osteoarthritis is associated with knee osteoarthritis. Conclusions based on mechanical axis radiographs. Arch Orthop Trauma Surg. 2008;128(6):555-60.

16. Muehleman C, Margulis A, Bae WC, Masuda K. Relationship between knee and ankle degeneration in a population of organ donors. Bmc Medicine. 2010;8(1):1-11.

17. Kamaruzaman H, Kinghorn P, Oppong R. Cost-effectiveness of surgical interventions for the management of osteoarthritis: a systematic review of the literature. BMC Musculoskelet Disord. 2017;18(1):183.

18. Franklin PD, Lewallen D, Bozic K, Hallstrom B, Jiranek W, Ayers DC. Implementation of patient-reported outcome measures in U.S. Total joint replacement registries: rationale, status, and plans. J Bone Joint Surg Am. 2014;96(Suppl 1):104-9.

19. Bourne RB, Chesworth BM, Davis AM, Mahomed NN, Charron KD. Patient satisfaction after total knee arthroplasty: who is satisfied and who is not? Clin Orthop Relat Res. 2010;468(1):57-63.

20. Kamenaga T, Muratsu H, Kanda Y, Miya H, Kuroda R, Matsumoto T. The influence of postoperative knee stability on patient satisfaction in cruciateretaining total knee arthroplasty. J Arthroplasty. 2018:33(8):2475-9.

21. Lange JK, Lee YY, Spiro SK, Haas SB. Satisfaction rates and quality of life changes following total knee arthroplasty in age-differentiated cohorts. J Arthroplasty. 2018;33(5):1373-8.

\section{Ready to submit your research? Choose BMC and benefit from:}

- fast, convenient online submission

- thorough peer review by experienced researchers in your field

- rapid publication on acceptance

- support for research data, including large and complex data types

- gold Open Access which fosters wider collaboration and increased citations

- maximum visibility for your research: over $100 \mathrm{M}$ website views per year

At $\mathrm{BMC}$, research is always in progress.

Learn more biomedcentral.com/submissions 